\title{
THE REAL-TIME MONITORING SERVICE PLATFORM FOR LAND SUPERVISION BASED ON CLOUD INTEGRATION
}

\author{
Sun Jiuhu ${ }^{1}$, Mao Jijun ${ }^{1}$, Xiang Hengmao ${ }^{1}$, Wang Gang ${ }^{2}$, Liang Yucai ${ }^{1}$ \\ ${ }^{1 .}$ Shandong Provincial Institute of Land Surveying and Mapping, 250102, Jinan, Shandong, China - sunjhgis@ 126.com \\ 2. Jinan Bureau of State Land Supervision Administration, 250014, Jinan, Shandong, China - 34355205@qq.com
}

Commission III, WG III/7

KEY WORDS: Land Supervision; Remote Sensing; Cloud Integration; Real-time Monitoring; Service Platform

\begin{abstract}
:
Remote sensing monitoring has become the important means for land and resources departments to strengthen supervision. Aiming at the problems of low monitoring frequency and poor data currency in current remote sensing monitoring, this paper researched and developed the cloud-integrated real-time monitoring service platform for land supervision which enhanced the monitoring frequency by acquiring the domestic satellite image data overall and accelerated the remote sensing image data processing efficiency by exploiting the intelligent dynamic processing technology of multi-source images. Through the pilot application in Jinan Bureau of State Land Supervision, it has been proved that the real-time monitoring technical method for land supervision is feasible. In addition, the functions of real-time monitoring and early warning are carried out on illegal land use, permanent basic farmland protection and boundary breakthrough in urban development. The application has achieved remarkable results.
\end{abstract}

\section{INTRODUCTION}

China established the State Land Supervision System in 2006. After continuous exploration by the Land Supervision Agencies of "Nine Bureau and One Office," a business system centered on daily supervision, routine supervision and special supervision has been formed (Qiu, 2016). The land supervision plays a great role in the protection of cultivated land, also in saving and intensive land use in China. The traditional land supervision methods are difficult to take on a wide range and full coverage of supervision tasks by using the methods of "human sight and field survey". The remote sensing methods provide a great technical support for land supervision and play an important role in improving the effectiveness and deterrence of land supervision, which could effectively reconcile conflicts between land supervision staff shortages and vast expanse supervision areas (Luo et al, 2010). In 2014, the Ministry of Land and Resources started the construction of an all-weather remote sensing monitoring system for land resources, focusing on two red lines of permanent basic farmland and urban development boundaries and gradually exploring all-round monitoring and regulation modes of land resources. However, the current remote sensing methods for land supervision are still based on regular distribution of monitoring patches which can't meet the requirements of real-time monitoring because of low timeliness and efficiency in problems detection.

In response to this problem, this paper designed an intelligent dynamic processing technology based on multi-source images and developed a cloud-integrated real-time monitoring service platform for land supervision.

\section{OVERALL TECHNICAL IDEA}

At present, the applications of domestic Gaofen series satellites and resource satellites are becoming more and more widespread. The high time resolution of image data and large-scale imaging make it possible for real-time land supervision in various provinces and cities. In view of the business needs, this paper explored and established a real-time monitoring technology system for land supervision and stepped up routine supervision to carry out real-time reminders and early warnings so as to enhance the effectiveness of supervision.

\subsection{The overall acquisition of remote sensing image data}

Currently, China has established a series of satellites of resources, meteorology, oceans, environment and disaster reduction and has initially formed a nation-to-land observing system, also has carried out different applications in such fields as land resources, ecological environment, meteorology and disaster reduction (Tang and Cong, 2011). According to the needs of land management, this paper selects civil highresolution optical satellite, including Gaofen-1, Gaofen-2 and resource 3-02 satellite, whose spatial resolution are about 1-2 meters. The lower spatial resolution of Gaofen-2 satellite can even reach 0.8 meters. Regardless of the weather conditions, the coverage of provincial-level administrative regions can be achieved once a month by taking advantages of abovementioned satellite data synthetically. As shown in the figure below, an effective image coverage formed by the blue frame after cloud detection covers the entire Shandong Province in mid to late November. Through the overall acquisition of the image data, it provides the basic data for the real-time monitoring of land management. 


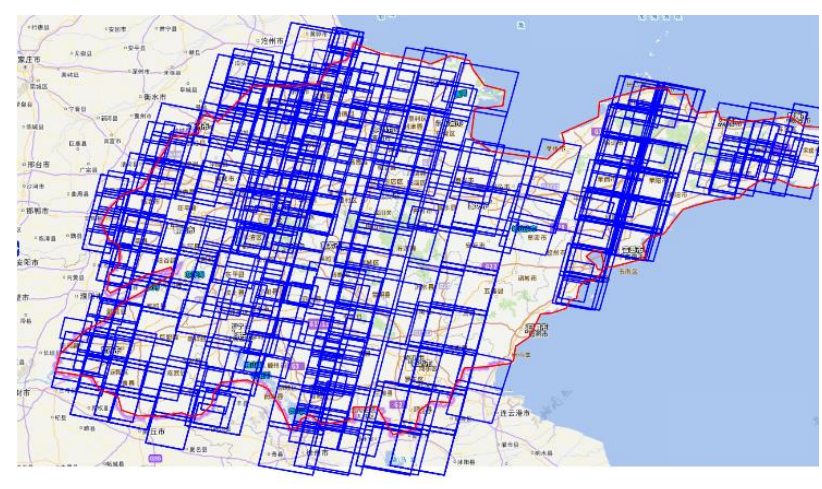

Figure 1. Satellite image coverage diagram

\subsection{Intelligent dynamic processing of multi-source image}

The traditional time-consuming and labor-consuming image processing methods can no longer meet the needs of mass image processing. Automation and rapid life-cycle management become the bottleneck restricting the application of remote sensing images (Mu et al, 2016). In this paper, a method of dynamic update of multi-source image orientation, correction and storage is put forward. Based on the RPC model image automatic registration technology, it achieves the automatic orientation, correction and storage of satellite images by means of orthographic image and DEM data. In addition, the technical process of dynamic update of data driven by changing information is constructed. The updated range of data is obtained automatically through the variation of data, and the updated image is replaced according to the updated range of intelligent matching, thereby providing the online service of the latest image to users. As shown in Figure 2.

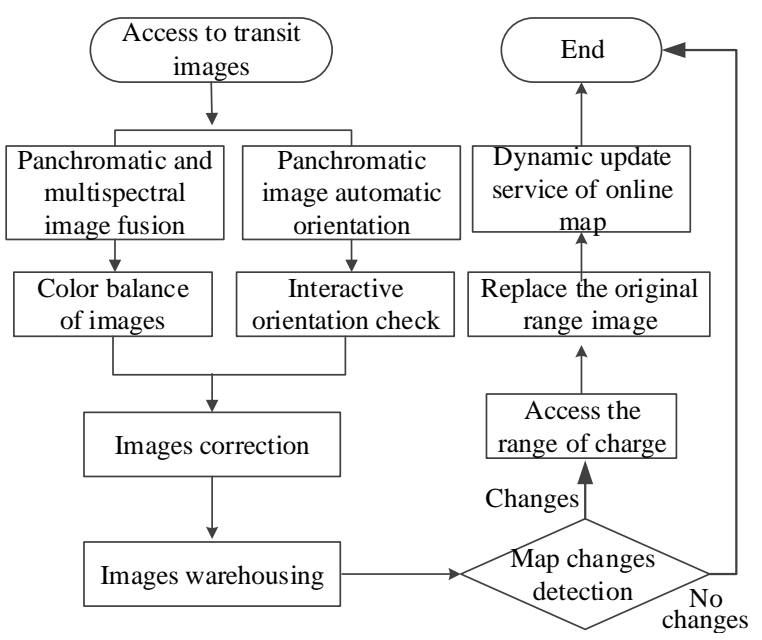

Figure 2. Flow chart of intelligent image processing technology

\subsection{The cloud-integrated real-time monitoring service platform for land supervision}

Geographic Information System is now facing the challenges of data intensive and spatiotemporal analyzing intensive. Cloud computing provides potential solutions for constructing high performance and high concurrency GIS due to its characteristics of resource pooling, on - demand self - service, and rapid elasticity (Ding et al, 2017). In this paper, the cloud-integrated real-time monitoring service platform for land supervision is developed. And the real-time monitoring service portal site is structured based on the B/S mode. Through the unified portal and other key technologies such as online collaboration, the monitoring services platform which integrates with real-time image services, reminders, early warning and decision-making analysis is also built. It has achieved multi-terminal interconnection, collaborative sharing of GIS application model and has created the service platform with the characteristics of normalization, real time, linkage between upper and lower.

\section{SYSTEM DESIGN}

This paper closely centers on the general request of Minister Jiang Daming to the State Land Agencies on "finding the problem at the beginning and resolving it in its infancy." By comprehensively using information support technologies such as remote sensing, geographic information system and cloud computing, we have constructed the real-time monitoring technology system and developed real-time monitoring service platform for land supervision.

\subsection{Overall structure}

Platform which takes use of SOA software architecture can be divided into support layer, data layer, business system layer and users layer, as shown in Figure 3. The operation of support layer relies on the existing infrastructure environment support platform and achieves the interconnection to supervision bureau, province, city, county and township through the Internet. The data layer includes the basic database of territorial resources, business database and monitoring image database. With the geographic information service provided by Map World as the uniform positioning datum, the data is distributed through the Web application server to provide Web data and functional services to the outside. The business system has established a business system integrating image services, patches check supervision, post-approval supervision and early warning, using and reporting at the same time with early warning, analysis briefings and field feedback APP as a whole, thus realizing "full area, full service and whole process "of the information system for land supervision. The users layer includes five layers like supervision bureau, province, municipality, county and township.

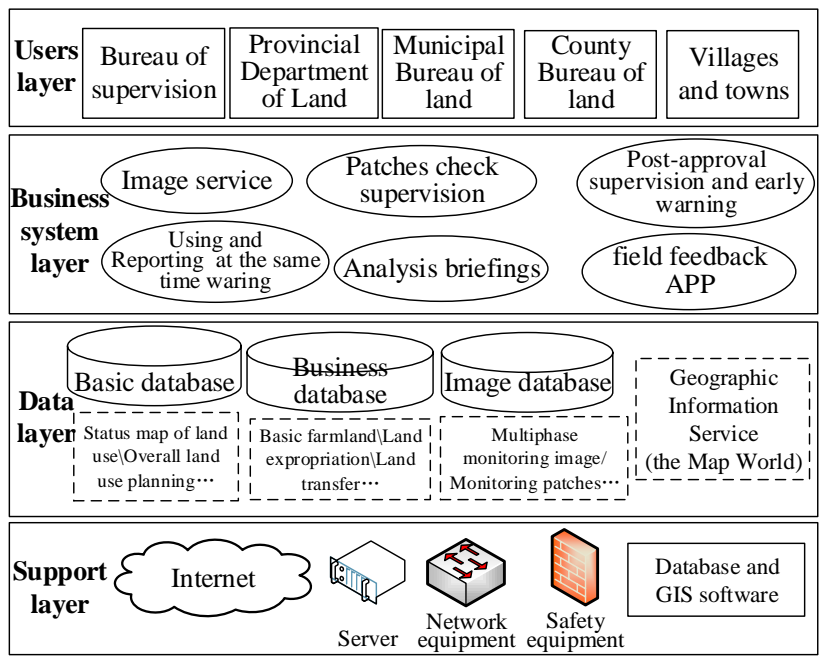

Figure 3. The overall architecture of platform 


\subsection{Data organization and management}

Based on the data type and application requirements, the vector data such as land supervision basic data and business data are used to establish the integrated database of land supervision through Oracle software, and the image data is stored by file.

3.2.1 Vector data: It uses the data type SDO_GEOMETRY (Wang, 2011) provided by Oracle Spatial for storage and establishes spatial index to optimize the retrieval performance of spatial data and improve the access efficiency to spatial data.

1) Through the integration and docking with the Ministry of Land Resources data center, it has established the land database which integrates land planning and "batch, supply, use, complement, check". And it can be divided into two categories of basic data and business data.

Basic data: The basic data refer to the basic and commonweal data generated from the survey and evaluation work, mainly including the data of land use status, remote sensing monitoring, urban cadastral, collective land ownership confirmation and urban datum price.

Business data: Business data refer to the data generated by the land administration and business management process. The data mainly include land business management database for construction land preliminary hearing, examination and approving constructive land, land expropriation, land supply, land remediation projects and cultivated land balance, land development and utilization, and supervision of law enforcement.

The above data are mainly used for discovering problems and comparison, and do not provide data services to outsiders.

2) Monitoring patch data. Based on satellite images, this paper monitors the situation of illegal land exploitation, the protection of permanent basic farmland and the breakthrough of urban development boundary in the supervision area, and forms the monitoring patch data. The publishing method of monitoring data is based on Map World in the paper (Sun et al, 2017), as shown in Figure 4.

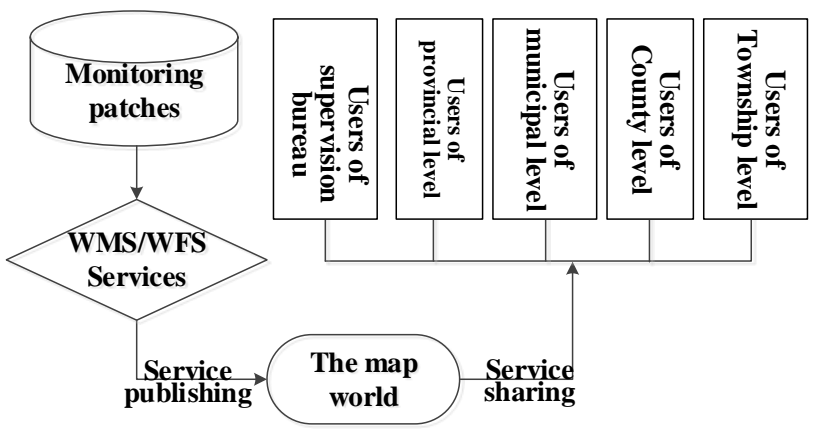

Figure 4 The monitoring data publishing based on the Map World
3.2.2 Image data: Image data is processed by intelligent dynamic processing of multi-source images. After RPC registration, the image results are stored in a mosaic data-set provided by ArcGIS (Huang et al, 2015), which provide image Web sharing services and support for $\mathrm{B} / \mathrm{S}$ structure access and mobile terminal access. The program uses a large number of real-time image processing technology to optimize the entire process. While ensuring the accuracy of data processing, it can significantly improve the image data production efficiency and save production process storage space and processing time consumption.

For the new transit image data, the platform supports direct publishing after pre-processing without pre-slicing. It achieves real-time publishing and view, also improves the timeliness of the image use. For the acquired transit data, it has been proved that platform can complete image data processing and sharing of the publishing in the same day.

\subsection{System functions}

3.3.1 Image Services: Image services are mainly oriented to grass-roots law enforcement officials, to provide data query, image patrol, clues verification, projects check and SMS notification.

1) Data query. The system provides real-time image services in accordance with the conditions of administrative divisions, collection time and satellite type. Therefore, it is convenient to keep abreast of image coverage situation in the surveillance areas, as shown in Figure 5.

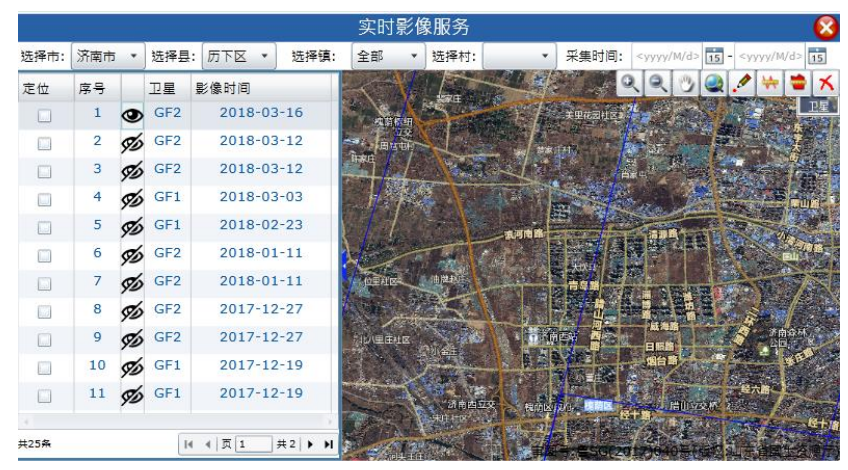

Figure 5. Data query function interface

2) Image patrol. Due to the shortage of front-line law enforcement officials and lack of equipment guarantee, dynamic patrol is difficult to cover all jurisdictions. As shown in Figure 6 , based on the image patrol function, the user can use the early and latter images to make double-screen comparison and find out the change patches and mark patches areas as well as basic information for the next field verification. 


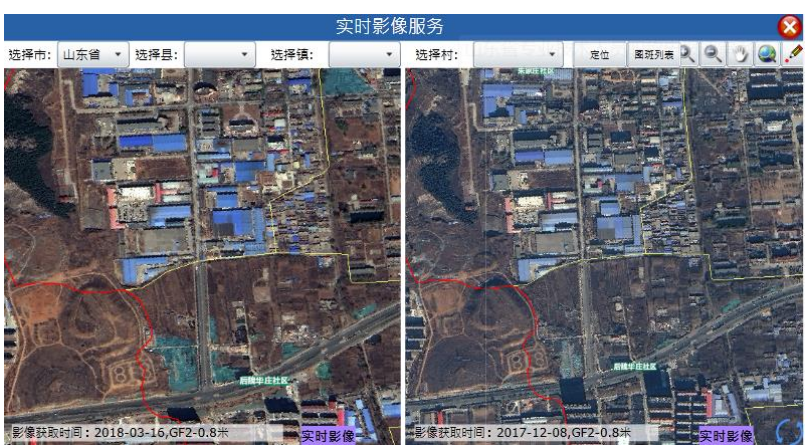

Figure 6. Image patrol function interface

3) Clues verification .The system integrates the administrative geographical names information which is detailed to the natural villages and is mainly used for the initial clues verification of letter visits, media exposure and leadership approval. By searching for clues such as place names and other location information, users can locate the corresponding location and view multiple images to understand the changes in the location of the land in order to verify the clues information.

4) Projects check. By uploading the standard format of the project site coordinates data, users can locate to the location of the block and view multiple images to understand the changes in the location of the land in order to assist decision management.

5) SMS notification. The system takes county as the basic unit. When the new transit image coverage beyond the jurisdiction of $10 \%$, it will send a SMS notification to the county administrator to inform the real-time image service updates and facilitate grass-roots personnel to work in a timely manner.

3.3.2 Monitoring patches supervision: Based on the images, the platform can supervise illegal land use of supervision area around the clock and detect the patches timely through in-house comparison. Then it will bring the patches into the system for unified management. Monitoring patches supervision provides functions for patches query and search, verification information report, check and identification and rectification and cancellation, as shown in Figure 7.

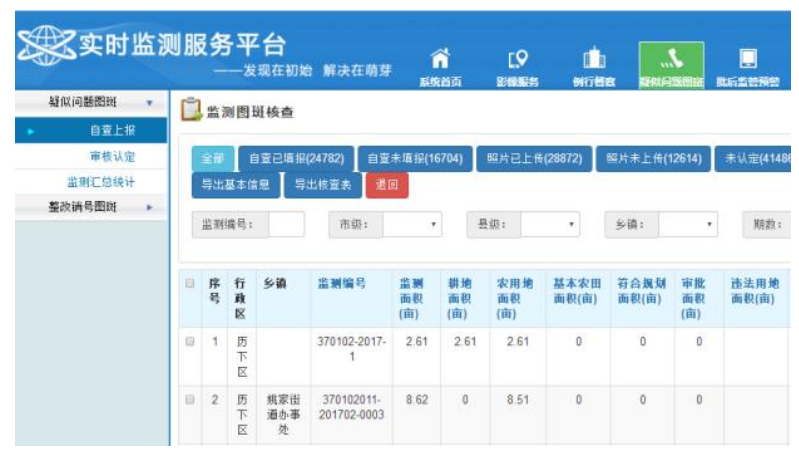

Figure 7. Monitoring patches supervision function interface
3.3.3 After-approval supervision and early warning: By using the land supplying record data and remote sensing images for comparison and analysis, the platform will remind warning to parcels that have not started or have been idle at the appointed time. And it will provide the functions of querying and searching as well as map locating information about the warning parcels.

3.3.4 Early warning to the situation of using and reporting at the same time: Based on the real-time image service, the supervision departments, when supervising the examination and approval of construction land at the provincial level, made preliminary warnings about the parcels where had been constructed at the approval but not take proper treatment to the person liable according to the latest remote sensing images.

3.3.5 Analysis briefings: In order to give full play to the main role of the government at all levels of land use management, the system regularly collects and analyzes the monitoring and early warning data to form visual charts and briefings in order to help the relevant leaders know the stage situation of land use management in the jurisdiction areas. As shown in Figure 8.

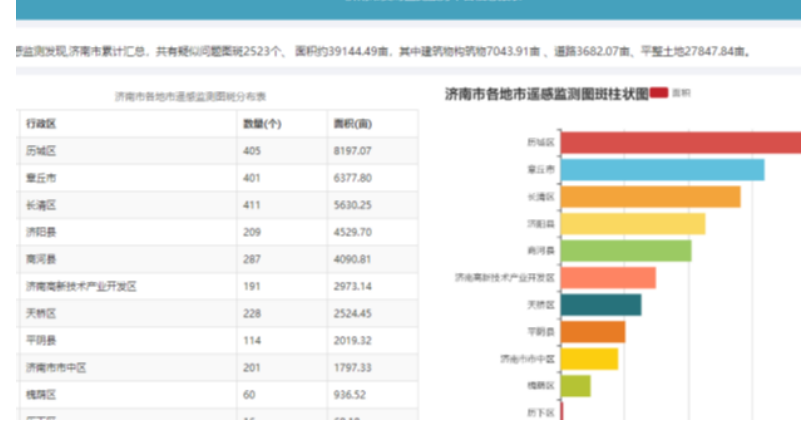

Figure 8. Analysis briefings function interface

3.3.6 Field feedback APP: In order to facilitate the grassroots officials to verify or rectify and cancel the accounts about the monitoring patches, this paper takes use of Android smart phones and tablet terminals to develop a field feedback APP which is based on mobile GIS technology. As shown in Figure 9. The system calls the electronic map data of Map World.

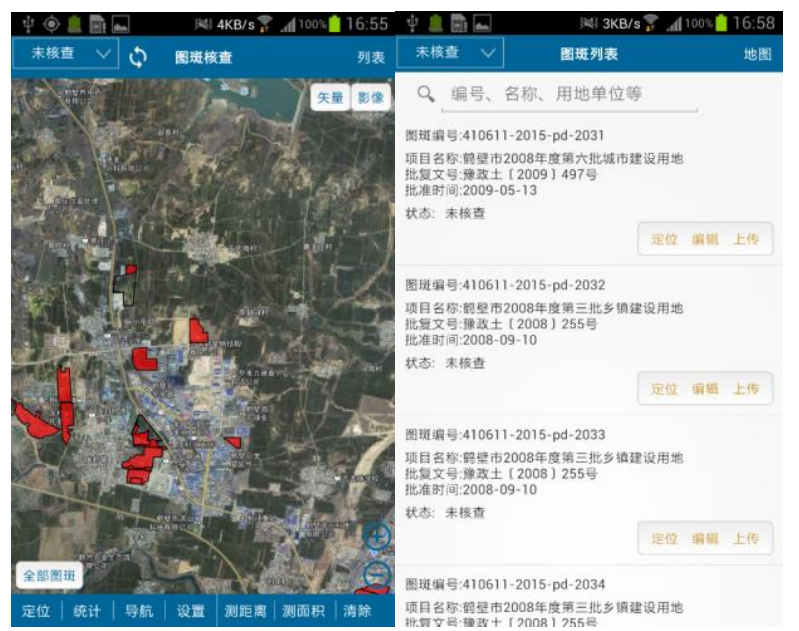

Figure 9. Field feedback APP interface 
Therefore the grass-roots officials can obtain the data of monitoring patches within the jurisdiction and send the information back to the system by locating the patches in the map through map navigation, also can check on the spot, take photos and define patches. The system will automatically record the photo coordinates, azimuth of taking photo, photographing time and other information, to improve the accuracy of the next determination.

\section{SYSTEM APPLICATIONS}

This paper designs a cloud-integrated real-time monitoring service platform for land supervision which is suitable for supervision bureau, provinces, cities, counties and towns. It was put into use in April 2017 by the Jinan Bureau of State Land Supervision, focusing on the situation of illegal land use, permanent farmland protection and urban exploitation boundary breakthroughs in the supervision areas, and carried out real-time monitoring and early warning for all levels of land management departments to provide real-time image services.

Since the launch of the platform, it has processed and published 1400 satellite images real-timely and the amount of data up to $4000 \mathrm{~GB}$. It can basically realize that "covering once a month" in most parts of Shandong and Henan provinces, and in Jinan, Zhengzhou, Qingdao and some other key cities can even obtain denser remote sensing images, to achieve 'covering once a halfmonth' (China Land Resources News, 2017). From the application situation, we found that most of the illegal use of land at the state of flat site or foundation in the two provinces and one city. Thus the extent of damage to the land is limited and investigation and rectification of small difficulties are conducive to the timely resolution of the problems. In accordance with before and in the course supervision principles, the platform can timely detect the problems of the post-grant supervision for land supplying and using and reporting at the same time for construction land. Practice has proved that the cloud-integrated real-time monitoring service platform for land supervision has established a new mode of land supervision that "looking by Map World, counting by Clouds and supervising by people" and truly achieved "early warning, early detection and early deterrence".

\section{CONCLUSIONS}

With the development of remote sensing and geographic information technology, land supervision departments should give full play to remote sensing technology as "Sky Eye". The multi-source image intelligent dynamic processing technology designed in this paper effectively improves the efficiency of large-scale image processing and improves the frequency of satellite image monitoring. Practice shows that the cloudintegrated real-time monitoring service platform for land supervision has carried out the monitoring of full-time relying on remote sensing images and built a normalized, real-time and upper-and-lower linkage service platform. In addition, the platform has practiced the demands of Minister Jiang Daming about the land supervision departments that "identify problems and solve problems both." Besides, it has improved the land supervision informatization level and helped local governments more timely and comprehensively grasp of the real situation to standardize the order of land management.
China Land Resources News, 2017, The construction of land monitoring service platform will help land supervision to innovate and develop, China Land Resources News, 2017-8$1(004)$

Ding, J.J., Su, L.L., Hu, Z.N., 2017, Research of Cloud GIS System Based on Cloud - terminal Integration Technology, Geomatics \& Spatial Information Technology, 40, pp. 51-55

Huang, Y., Dong, J., Ma, Y., et al, 2015, ArcGIS Based ZY3 Imagery Basemap Quick Processing Solution Research, Journal of Geo-Information Science, 17(12), pp. 1529-1535

Luo, Y.F., Xu, P.L., Li, Y.C., et al, 2010, The Application of 3 S Technology to Land Supervision, Remote Sensing for Land \& Resources, 22(3), pp. 12-15

Mu, L., Xie, G.X., Yang, R.J., et al. 2016, A Batch Processing Method Dealing with Massive Domestic High-Resolution Remote Sensing Satellite Image Data based on B/S Mode, Land and Resources Information, 1, pp. 22-26

Qiu, D.H., 2016, Primary Investigation of Core Business Innovation of Land Supervision, Land and Resources Information, (6), pp. 27-30

Sun, J.H., Xiang, H.M., Li, H., et al, 2017, Research on Data Release and Verification Method of Mine Remote Sensing Monitoring, Land and Resources Information, (1), pp. 26-29

Tang, X.M., Cong, N., 2011, Present Situation and Development of Surveying and Mapping Satellites China, Geomatics World, (2), pp. 40-44

Wang, Y.F., 2011, On the Application of Oracle Spatial Data Memory Management Technique, Bulletin of Surveying and Mapping, (6), pp. 6-79

\section{REFERENCES}

